\title{
Heisenberg and Bethe Field Extensions Applied to Magnetic Rings
}

\author{
G. BAnAszaK ${ }^{a, *}$, D. Blinkiewicz ${ }^{a}, \mathrm{P} . \mathrm{Krason}^{b}, \mathrm{~J} \mathrm{Milewski}^{c}$ \\ ${ }^{a}$ Faculty of Mathematics and Computer Science, Adam Mickiewicz University, \\ Umultowska 87, 61-614 Poznań, Poland \\ ${ }^{b}$ Department of Mathematics and Physics, Szczecin University, Wielkopolska 15, 70-415 Szczecin, Poland \\ ${ }^{c}$ Institute of Mathematics, Poznań University of Technology, Piotrowo 3A, 60-965 Poznań, Poland \\ We consider striking connections between the theory of homogenous isotropic Heisenberg ring (XXX-model) \\ and algebraic number theory. We explain the nature of these connections especially applications of Galois theory for \\ computation of the spectrum of the Heisenberg operators and Bethe parameters. The solutions of the Heisenberg \\ eigenproblem and Bethe Ansatz generate interesting families of algebraic number fields. Galois theory yields \\ additional symmetries which not only simplify the analysis of the model but may lead to new applications and \\ horizons.
}

DOI: 10.12693/APhysPolA.133.441

PACS/topics: 02.10.De, 03.65, 73.21-b, 75.10.Jm, 85.35Be, 89.70Be

\section{Introduction}

For the last 10 years a new method of investigating Quantum Mechanics in the context of Bethe Ansatz for the magnetic homogeneous ring has been introduced in the papers [1-4]. The idea of this new method is based on the observation that the Heisenberg Hamiltonian is an arithmetic operator. We define an arithmetic operator as follows.

Definition 1.1 (Arithmetic Operator) Let $V$ be a finite dimensional vector space over $\mathbb{C}$. Let $A: V \rightarrow V$ be $a \mathbb{C}$ linear operator. $A$ is called arithmetic operator if there is a lattice $\Lambda \subset V$ and a $\mathbb{Z}$-linear operator $A_{0}: \Lambda \rightarrow \Lambda$ such that $A_{0} \otimes_{\mathbb{Z}} \mathbb{C}=A$.

The spectrum of an arithmetic operator consists of algebraic numbers hence one can apply algebraic number theory and Galois theory to investigate it.

The natural arithmetic structure on Heisenberg Hamiltonians can be seen in the following way. The Hilbert space of quantum states for the Heisenberg ring with $N$ nodes is given by the $N$-th tensor power

$$
\mathcal{H}:=\mathcal{H}_{N}:=\hat{h}^{\otimes N}
$$

of the single-node two-dimensional Hilbert space $\hat{h}:=L_{\mathbb{C}}\{|0\rangle,|1\rangle\}$, where $|1\rangle$ (resp. $|0\rangle$ ) means that there is (resp. there is no) spin deviation at the node, and $L_{\mathbb{C}}\{$.$\} denotes the linear span of the set \{$.$\} over$ the field $\mathbb{C}$. The Heisenberg Hamiltonian $\hat{H}$ with the nearest neighbour interactions in the basis of magnetic configurations for the $N$-gone magnetic ring is defined as follows:

$$
\hat{H}: \mathcal{H} \rightarrow \mathcal{H} \quad \hat{H}:=\sum_{j \in \mathbb{Z}_{N}}\left(\tau_{j}-\mathrm{id}_{\mathcal{H}}\right)
$$

*corresponding author; e-mail: banaszak@amu.edu.pl where $\tau_{j}$ denotes the transposition of $j$-th and $(j+1)$ th $(\bmod N)$ factors in the tensor power $\hat{h}^{\otimes^{N}}$. The investigation of this operator in relation with the famous Bethe Ansatz attracted attention of many mathematicians and physicists eg. $[5,6]$, (combinatorial approach to Bethe Ansatz) [7,8] (algebraic approach to Bethe Ansatz). Combinatorics of Bethe Ansatz was also an inspiration for finding some new relations among coefficients of Gaussian polynomials [9].

\section{Arithmetic approach to Heisenberg Hamiltonian}

In our approach to the Heisenberg Hamiltonian and Bethe Ansatz [1-4, 10] we took advantage of the following observation we had made. Namely, let $\mathcal{H}_{\mathbb{Z}}:=\mathcal{H}_{\mathbb{Z}, N}:=$ $h^{\otimes N}$ be the $N$-th tensor power of the rank 2 , free $\mathbb{Z}$ module $h:=L_{\mathbb{Z}}\{|0\rangle,|1\rangle\}$, where $L_{\mathbb{Z}}\{$.$\} denotes the linear$ span of the set $\{$.$\} over the ring of integers \mathbb{Z}$. Then one can define the operator:

$$
H: \mathcal{H}_{\mathbb{Z}} \rightarrow \mathcal{H}_{\mathbb{Z}}, \quad H:=\sum_{j \in \mathbb{Z}_{N}}\left(\tau_{j}-\mathrm{id}_{\mathcal{H}_{\mathbb{Z}}}\right) .
$$

Hence $\hat{H}=H \otimes_{\mathbb{Z}} \mathbb{C}$ is the complexification of $H$ and clearly $\hat{H}$ is an arithmetic operator. It is thus natural to study the Galois symmetry of various parameters related to the spectrum and eigenspaces of the Heisenberg Hamiltonian cf. Fig. 1.

Next, we consider the extension $\mathbb{Q}\left(\xi_{N}\right) / \mathbb{Q}$ and apply the discrete Fourier transform to change the basis of the configuration space. This yields a decomposition of the Heisenberg operator (1) into blocks.

The natural basis of (1) corresponds to the $\mathbb{C}$-span of the space $Q_{r}$ of $r$ deviations $(1 \leqslant r \leqslant N)$ and thus

$$
\mathcal{H}=\bigoplus_{r=1}^{N} \mathcal{H}_{r}, \quad \mathcal{H}_{r}=\mathbb{C} \otimes_{\mathbb{Z}} \operatorname{Span}_{\mathbb{Z}} Q_{r} .
$$

Notice that $\mathbf{j} \in Q_{r}$ is represented by a sequence

$$
\mathbf{j}=\left(j_{1}, j_{2}, \ldots, j_{r}\right), \quad 1 \leqslant j_{1}<j_{2}<\cdots<j_{r} \leqslant N
$$

The spaces $\mathcal{H}_{r}$ are invariant with respect to 


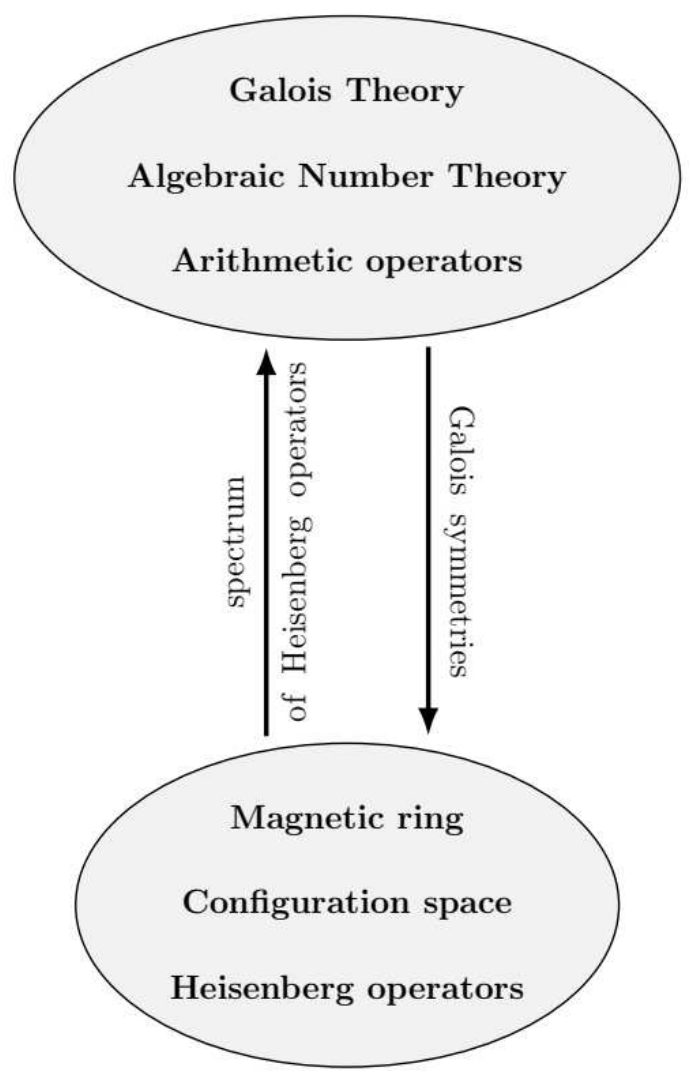

Fig. 1. Diagram of mutual connections.

the Heisenberg operator (2). Let

$$
\hat{S}^{-}: \mathcal{H}_{r} \rightarrow \mathcal{H}_{r+1}
$$

be the step operator which in the "ket" notation has the following values on the basis elements

$$
\hat{S}^{-}\left|\left\{j_{1}, \ldots, j_{r}\right\}\right\rangle=\sum_{j \notin\left\{j_{1}, \ldots j_{r}\right\}}\left|\left\{j, j_{1}, \ldots, j_{r}\right\}\right\rangle .
$$

We have

$$
\hat{S}^{-} H_{r}=H_{r+1} \hat{S}^{-}
$$

This allows one to define the subspace of highest weight $\mathcal{H}_{r, r}$ to be the subspace orthogonal to the image of $\hat{S}^{-}$on $\mathcal{H}_{r}$ and for $r^{\prime}<r$ the subspaces $\mathcal{H}_{r, r^{\prime}}:=\left(\hat{S}^{-}\right)^{r-r^{\prime}} \mathcal{H}_{r^{\prime}, r^{\prime}}$. After introducing relative positions of the Bethe pseudoparticles:

$$
\boldsymbol{t}=\left(t_{1}, \ldots, t_{r}\right), \quad t_{\alpha}=\left\{\begin{array}{l}
j_{\alpha+1}-j_{\alpha}, \quad 1 \leqslant \alpha<r \\
N+j_{1}-j_{r}, \quad \alpha=r
\end{array}\right.
$$

we can identify the states which are in the same orbit of the action of the cyclic group $C_{N}$ :

$$
\left(t_{1}, \ldots, t_{r}\right) \sim\left(t_{2}, t_{3}, \ldots, t_{1}\right) \cdots \sim\left(t_{r}, t_{1}, \ldots, t_{r-1}\right)(10)
$$

Now the Fourier transform on each orbit, except of vacuum $(\mathrm{r}=0)$ and antivacuum $(\mathrm{r}=\mathrm{N})$ is given by the following formula

$$
|B, r, \boldsymbol{t}, k\rangle=\sum_{j \in \tilde{N}} F_{j k}|Q, r, \boldsymbol{t}, j\rangle, \quad k \in B
$$

where $B$ is the Brillouin zone for the ring,
$B:=\{-(N-1) / 2 \leqslant k \leqslant(N-1) / 2\}$ for $N$ odd and $B:=\{0, \pm 1, \ldots \pm(N / 2-1), N / 2\}$ for $N$ even. $F_{j k}=\omega_{N}^{-k j}, \tilde{N}=\{1,2, \ldots, N\}$. Finally, $|Q, r, \boldsymbol{t}, j\rangle$ denotes the arithmetic basis of the space of $C_{N}$ orbits in $\mathcal{H}$.

The new basis $|B, r, \boldsymbol{t}, k\rangle$ called the normalized basis of wavelets yields the following $C_{N}$-invariant decomposition

$$
\mathcal{H}_{r}=\bigoplus_{k \in B} \mathcal{H}_{r}^{k}
$$

where $\mathcal{H}_{r}^{k}$ is a subspace with a fixed quasimomentum $k$. In this setting one obtains further decomposition

$$
\mathcal{H}_{r}^{k}=\bigoplus_{r^{\prime}} \mathcal{H}_{r, r^{\prime}}^{k}, \quad \mathcal{H}_{r, r^{\prime}}^{k}:=\mathcal{H}_{r, r^{\prime}} \cap \mathcal{H}_{r}^{k}
$$

Thus we see that for any $N$ we have obtained a block decomposition of the Heisenberg operator (1). The dimensions of these blocks are considerably smaller than the dimension of the original operator and it is easier to compute eigen polynomials therefore also eigenvalues and eigenspaces for them. The diagram of relevant fields involved in the diagonalization of the Heisenberg arithmetic operator is depicted in Fig. 2. We describe them now. $\mathbb{Q}(\omega)$ and $\mathbb{Q}\left(\omega^{k}\right)$ are cyclotomic extensions of rationals $\mathbb{Q}$. The fields $\mathbb{Q}(\omega+\bar{\omega})$ and $\mathbb{Q}\left(\omega^{k}+\bar{\omega}^{k}\right)$ are their maximal real subfields. Decomposition of the space $\mathcal{H}$ into the invariant subspaces

$$
\mathcal{H}=\bigoplus_{r, r^{\prime}, k} \mathcal{H}_{r, r^{\prime}}^{k}
$$

in the normalized basis of wavelets yields Heisenberg fields $\mathcal{F}_{\mathbb{R}} H_{r, r^{\prime}}^{k}$ which are the extensions of the totally real fields $\mathbb{Q}\left(\omega^{k}+\bar{\omega}^{k}\right)$ by the eigenenergies of the Heisenberg operator on the subspace $\mathcal{H}_{r, r^{\prime}}^{k}$. The field $\mathcal{F}_{\mathbb{R}} \mathcal{H}_{r}^{k}$ is the following composition (compositum) of fields in $\overline{\mathbb{Q}}$ :

$$
\mathcal{F}_{\mathbb{R}} \mathcal{H}_{r}^{k}:=\bigvee_{r^{\prime}} \mathcal{F}_{\mathbb{R}} \mathcal{H}_{r, r^{\prime}}^{k}
$$

and similarly

$$
\mathcal{F}_{\mathbb{R}} \mathcal{H}_{r}:=\bigvee_{k \in B} \mathcal{F}_{\mathbb{R}} \mathcal{H}_{r}^{k}
$$

and

$$
\mathcal{F}_{\mathbb{R}} \mathcal{H}:=\bigvee_{r} \mathcal{F}_{\mathbb{R}} \mathcal{H}_{r}
$$

The corresponding complex Heisenberg fields are minimal extensions that contain appropriate real Heisenberg fields and the cyclotomic field. Thus e.g.

$$
\mathcal{F} \mathcal{H}^{k}:=\mathcal{F}_{\mathbb{R}} \mathcal{H}^{k} \mathbb{Q}\left(\omega^{k}\right)
$$

and

$$
\mathcal{F H}:=\mathcal{F}_{\mathbb{R}} \mathcal{H} \mathbb{Q}(\omega)
$$

The Bethe fields $\mathcal{B}^{k}$ and $\mathcal{B}$ resp. are extensions of $\mathcal{F} \mathcal{H}^{k}$ and $\mathcal{F H}$ resp. by the nonsingular Bethe parameters, which are solutions of Bethe system of equations [11]

$$
\begin{aligned}
a_{\alpha}^{N} & =\prod_{\beta \in \widetilde{r} \backslash\{\alpha\}}\left(-\frac{a_{\alpha} a_{\beta}-2 a_{\alpha}+1}{a_{\alpha} a_{\beta}-2 a_{\beta}+1}\right), \\
\alpha & =1, \ldots, r,
\end{aligned}
$$

where $\widetilde{n}:=\{1, \ldots, n\}$. The complex Heisenberg field is generated by

$$
\omega^{-k}=\prod_{\alpha=1}^{r} a_{\alpha}, \quad \text { and } \quad \widetilde{E}=\sum_{\alpha=1}^{r}\left(a_{\alpha}+a_{\alpha}^{-1}\right),
$$

where the first quantity corresponds to a quasi- 
momentum and the second to shifted energy. The assumption that all parameters are nonzero is essential and yields the highest weight solutions $\left(r^{\prime}=r\right)$. Solutions that differ up to an order of parameters are viewed as the same. The Bethe field $\mathcal{B}^{k}$ is the extension of $\mathbb{Q}$ by the solutions of Bethe equations with fixed $k$ i.e. the product of parameters is equal to $\omega^{-k}$. Computations of Galois groups for the extensions in Fig. 2 enable us to obtain better understanding of Galois symmetries of the physical system coming from the Heisenberg ring and Heisenberg Hamiltonian.

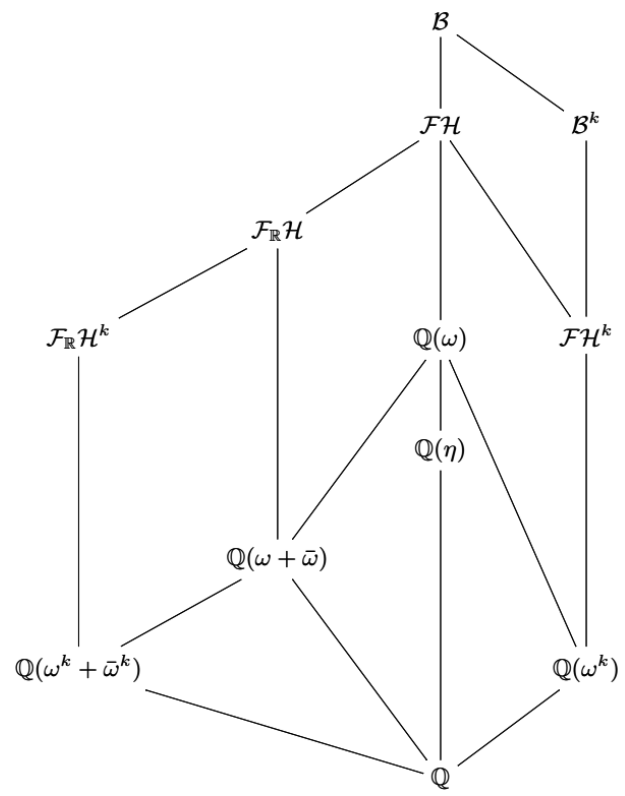

Fig. 2. Diagram of fields. $\omega:=\zeta_{N}, \eta=\zeta_{q}$ are primitive roots of unity. Let $p$ be a prime number. Then $q=p$ if $p \mid N$ and $p \neq 2$ or $q=4$ if $N=2^{s}$ and $s>1$.

\section{Acknowledgments}

JM was partially supported by the grant 04/43 PS PB /0087 of Ministry of Science and Higher Education of Poland.

\section{References}

[1] G. Banaszak, S. Barańczuk, T. Lulek, J. Milewski, R. Stagraczyński, Acta Phys. Pol. A A.121, 1111 (2012).

[2] J. Milewski, G. Banaszak, T. Lulek, M. Łabuz, R. Stagraczyński, Open Syst. Inf. Dyn. 19, 1250012 (2012).

[3] G. Banaszak, B. Lulek, T. Lulek, J. Milewski, B. Szydło, Rep. Math. Phys. 71, 205 (2013).

[4] J. Milewski, Rep. Math. Phys. 70, 345 (2012).

[5] S.V. Kerov, A.N. Kirillov, N.Y. Reshetikhin, J. Sov. Math. 41, 916 (1988).

[6] A.N. Kirillov, N.Y. Reshetikhin, J. Sov. Math. 41, 925 (1988).

[7] R. Langlands, Y. Saint-Aubin, Aspects combinatoires des équations de Bethe, First appeared in Adv. Math. Sci.: CRM's 25 years, Ed. L. Vinet, CRM Proc. and Lecture Notes, Am. Math. Soc., 1997.

[8] R. Langlands, Y. Saint-Aubin, Algebro-geometric aspects of the Bethe equations, Proc. of Gürsey Memorial Conference, Springer-Verlag 1995.

[9] P. Krasoń, J. Milewski, Cyclic group actions and restricted partitions, preprint (2017).

[10] J. Milewski, G. Banaszak, T. Lulek, Open Syst. Inf. Dyn. 17, 89 (2010).

[11] H. Bethe: Z. Physik 71, 205 (1931). 